\title{
The Role of Intensional and Extensional Interpretation in Semantic Representations - The Intensional and Preextensional Layers in MultiNet
}

\author{
Hermann Helbig and Ingo Glöckner \\ University at Hagen, Intelligent Information and Communication Systems, Germany \\ \{hermann.helbig/ingo.gloeckner\} @fernuni-hagen.de
}

\begin{abstract}
Although it is well known that the full meaning of a concept includes an intensional and an extensional aspect, the latter is neglected in almost all practically used knowledge representations and semantic formalisms, which consider only the interrelationships between concepts but not their referential meaning. In logically oriented representations with the usual model-theoretic interpretation, the extensions of predicates or symbols of the language in general belong to a metalevel (the model level) clearly distinguished from the logical language. In this paper, we show in what way the extensional aspect is important (in addition to the intensional aspect) to account for the full meaning of natural language expressions. The necessity of dealing with both types of meaning is illustrated by means of semantic phenomena like factual and intensional negation, intensional quantification and cardinalities, or by the meaning of prepositions describing set relationships, and others. We will utilize the framework of Multilayered Extended Semantic Networks (MultiNet) to discuss these issues. The MultiNet formalism is successfully used in various NLP applications. Its explicit modeling of intensional and preextensional layers offers an explanation of the interplay of intension and extension of conceptual entities in the overall process of constituting the meaning representations of natural language expressions.
\end{abstract}

\section{Introduction}

At the latest since Carnap, it is well known that the full meaning of a concept is constituted by two aspects, its intension and its extension [1]. The first part comprises the embedding of a concept in the world of concepts as a whole, i.e. the totality of all relations to other concepts. The second part establishes the referential meaning of the concept, i.e. its counterpart in the real or in a possible world. The intension of the concept Napoleon I, for instance, comprises the relations to the concepts France, emperor, etc., while its extension is a concrete historical person. The concept emperor again is related to government, autocracy, etc., while its extension is the set of all emperors of this world. The aim of this paper is to show the necessity to deal with both aspects in a knowledge representation and to discuss to what degree this can really be done.

Of course, it is impossible to cope with the full extensions of concepts on a computer, since some concepts have an infinite extension (e.g. successor), some have a fuzzy extension (e.g. hill, large), and some (like extension) arguably cannot be assigned 
an extension at all. These observations suggest that one should rather incorporate partially described extensions in a KRS ${ }^{1}$, which represent only typical members or subsets of the extension of the concept, or only the cardinalities of the corresponding sets. Thus, we speak of a "preextensional" instead of an "extensional" interpretation. It is postulated that the extensional meaning of a concept has to be modeled in the representational formalism itself (not outside of it as in logic-oriented KRS with a model-theoretic semantics). The role of intension and extension in the semantic interpretation of linguistic expressions and even in coreference resolution, is illustrated by the following example: "(A group of $\left.\left.(\text { four men })_{[\mathrm{CARD}=4]}\right)\right)_{[\mathrm{CARD}=1]}$ found $(\text { many gems })_{i}$. Six of $\left(\text { them }_{i}\right)_{[\mathrm{CARD}>6]}$ had been stolen."

The example shows the following: Semantically, there is one group of men, not several groups (as indicated by $[\mathrm{CARD}=1]$ ), consisting of four men (indicated by $[\mathrm{CARD}=4]$ ), who discovered a rather underspecified set of gems. Nobody would figure out during understanding of the first sentence how many gems there are (or any other details on the extension of the concept described by many gems). But, when reading the second sentence, it becomes clear from the partitive construction six of them that the pronoun them cannot refer to the group of men (since there are only four) but must rather be linked to the meaning of the phrase many gems, indicated by the index $i$. Moreover, the preposition of in the phrase four of them establishes a set inclusion between the extensions of the conceptual entities denoted by many gems and the stolen objects. It becomes also clear that the set underlying the intensional meaning of the quantificationally underspecified phrase many gems must have a cardinality greater than 6 . All this has to be modeled in a full meaning representation (see Sect. 2.1); the semantic representation of an analogous example is shown in Figure 4.

There are few proposals to deal with this extensional dimension besides the intensional aspect in a KRS, one early example is [2]. Elements of the extensional meaning of concepts are also present in KL-ONE [3] and its successors, i.e. in several kinds of Description Logics [4]. Here, we meet them in form of the cardinalities of roles. The aspect of extensionality which traditionally raised most interest is that of characterizing the real-world existence or facticity of entities mentioned in a discourse. Apart from the vast literature on presuppositions, this topic has also been addressed in modal logics [5] and in the context of knowledge representation [6-9].

In the paper, we discuss these issues from the perspective of Multilayered Extended Semantic Networks (MultiNet), a KRS designed with a clear distinction of an intensional layer and a preextensional layer in mind. The formalism, extensively documented in [10], is successfully used in large NLP applications, ranging from question answering over natural language interfaces to electronic teaching (see Sect. 8). After introducing the core concepts of MultiNet, we turn to the main topic, discussing several phenomena of linguistic relevance whose treatment in a KRS calls for a modeling on a (pre)extensional level. We address the distinction of intensional quantification vs. preextensional cardinality, the discernment of intensional and preextensional negation, treatment of existential presuppositions and of implicative or factive verbs, the modeling of counterfactuals, and the modeling of set relationships implicit in partitive constructions and quantification involving coordinations.

\footnotetext{
${ }^{1}$ Knowledge Representation System(s)
} 


\section{Multilayered Extended Semantic Networks}

\subsection{The Main Characteristics}

In designing a KRS, one should first specify a set of criteria to be satisfied by the envisioned knowledge representation. In our context, the most important of these criteria are: 1) cognitive adequacy - which to the very least means that the semantic representation should be concept-centered in the sense that every conceptual entity has a unique representative; 2) homogeneity - the descriptive means should be suitable for defining the semantics of lexemes, the semantics of sentences or texts, and the axiomatic apparatus and inference rules of the KRS as well; and 3) stratification - it must be possible to represent the different semantic aspects (like intensional vs. extensional aspects, or immanent vs. situational aspects of meaning) in different layers of the KRS.

Like other semantic network formalisms, MultiNet represents conceptual entities by nodes, while relations between concepts are represented as arcs between these nodes (see Figure 4). However, MultiNet has several additional features:

1. The nodes of the SN are classified according to a predefined conceptual ontology, i.e. by a sort assignment.

2. The nodes have a rich inner structure, i.e. the relevant classificatory dimensions of conceptual entities are expressed by values of 'layer attributes' of each node.

3. The formalism commits to a fixed set of relations in order to provide a stable basis for axiomatization and a platform for long-term projects like lexicon development.

4. The distinction of an intensional layer and a preextensional layer has already been mentioned. Each layer comes with its own relations and functions and its own layer attributes which refine the description of a node at the considered level.

5. We discern categorical knowledge about a concept (e.g. for the concept car: "A car has a motor"), prototypical knowledge about the concept which only holds by default ("A car typically has an airbag") and finally so-called situational knowledge, i.e. contingent knowledge about a concept which is irrelevant with respect to the concept's core meaning. For example, the introduction of a specific car in the sentence "Paul bought a car" is situational with respect to the generic concept car and has no effect on the meaning of car in general.

6. Axiomatic rules (meaning postulates) are employed for interrelating the meanings of natural language expressions described in the lexicon. They also define the basic relations and functions, which can be viewed as nodes at a metalevel.

For a complete documentation of the expressional means of MultiNet see [10].

\subsection{Sorts and Features of concepts}

In MultiNet, every node is assigned a sort from a fixed sort hierarchy whose upper part is shown in Figure 1. The primary purpose of these sorts is that of defining the signatures (i.e. the domains and value restrictions) of the relations and functions provided by the formalism. The restrictions imposed by the sorts on the use of relations and functions or on the valency frames of verbs, nouns, and adjectives are automatically enforced by the workbenches for the knowledge engineer [11] and for the computer lexicographer 


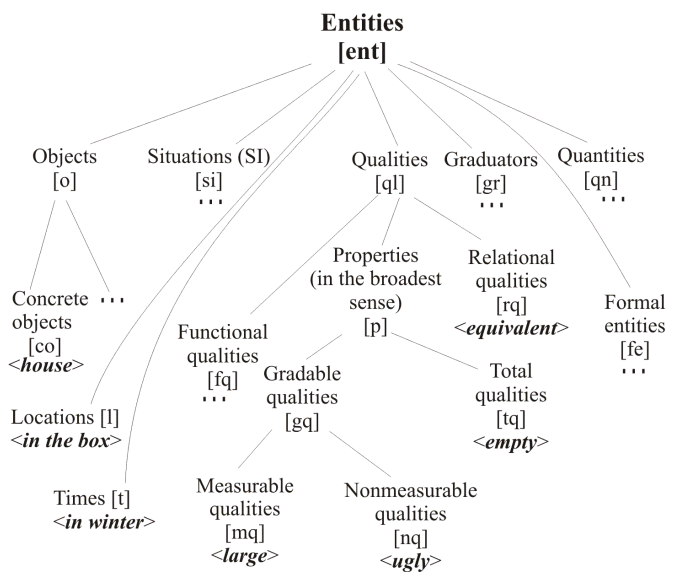

Fig. 1. The Upper Part of the Hierarchy of MultiNet Sorts

[12], which use MultiNet for representing knowledge and describing lexical semantics. The sorts are crucial for the formal definition of the representational means, but also an important source of information for disambiguation in the semantic interpretation of linguistic constructs (e.g. prepositional phrases). However, the sorts alone are too coarse to specify arbitrary selectional restrictions. For that one needs additional semantic features (e.g. being animated or being an artifact) also offered by MultiNet [12].

\subsection{Relations and Functions of MultiNet}

The formal devices for interlinking the conceptual nodes of a semantic network are relations and functions. MultiNet provides a fixed set of about 140 semantic relations and functions which can roughly be classified into two main groups.

Firstly, there are relations and functions of the intensional level. They are used to describe the inner structure of conceptual objects and situations as well as their relationships to other entities. Typical descriptions of objects involve the characterization of their material structure (by the part-whole-relationship PARS), of their material origin (described by the ORIGM relation), or of their properties (using the PROP relation). Situational concepts like events or states, on the other hand, are typically defined by specifying the participants involved in the situation and the semantic roles which characterize the relationship between a situation and its participants. Typical examples are deep case relations like AGT (agent), EXP (experiencer), or OBJ (neutral object). Additionally, situations are characterized by their spatio-temporal embedding (e.g. location LOC or temporal specification TEMP). See Table 1 for a brief description of the relations on the intensional level referenced in this paper.

Second, there are also relations and functions of the preextensional level (see Table 2). They offer the descriptive means needed for modeling sets and other extensional 
Table 1. Typical relations of the intensional layer. The notation $\ddot{o}$ requires an entity of sort $o$ with [ETYPE $>0$ ] (i.e. a plurality) while $\bar{o}$ or $\overline{s i}$ require generic concepts with $[\mathrm{GENER}=g e]$.

\begin{tabular}{lll}
\hline Relation & Signature & Short Characteristics \\
\hline AFF & $s i \times[o \cup s i]$ & Affected object \\
AGT & $s i \times c o$ & Agent \\
ANTE & {$[s i \cup t] \times[s i \cup t]$} & Temporal successorship \\
ASSOC & $e n t \times e n t$ & Association \\
ATTCH & $o \times o$ & Attachment of objects to objects \\
AVRT & $d y \times o$ & Aversion/Turning away \\
CAUS & $s i \times s i$ & Causality relation \\
COND & $s i \times s i$ & Conditional relation \\
EXP & $s i \times o$ & Experiencer \\
HSIT & $s i \times s i$ & Composition of situations \\
LOC & $c o \times l$ & Location of an object \\
MCONT & $s i \times[s i \cup o]$ & Mental or informational content \\
MODL & $s i \times m d$ & Modal restriction \\
OBJ & $s i \times[o \cup s i]$ & Neutral object \\
ORIGM & $c o \times c o$ & Relation of material origin \\
ORNT & $s i \times o$ & Orientation towards something \\
PARS & $c o \times c o$ & Part-whole relation for concrete objects \\
PRED & $\ddot{o} \times \bar{o}$ & Specification of a plurality \\
POSS & $o \times o$ & Possession \\
PROP & $o \times p$ & Relation between object and property \\
SUB & $o \times \bar{o}$ & Conceptual subordination (for objects) \\
SUBS & $s i \times \overline{s i}$ & Conceptual subordination (for situations) \\
SUBST & {$[o \times o] \cup[s i \times s i]$ Substitution/Replacement } \\
TEMP & $s i \times[s i \cup t]$ & Relation of temporal containment \\
\hline & &
\end{tabular}

representatives. Some rationale for introducing these constructs has already been given in the introduction and the remainder of the paper will focus on these constructions.

Relations and functions are characterized by implicational axioms familiar from first-order predicate logic. We discern two types of axioms: 1) B-axioms which interrelate natural language concepts; and 2) $R$-axioms which connect relations and functions of the formalism without reference to any lexicalized concept. The connection between the concepts kill and die is a typical case of a B-axiom:

$$
\left(s v_{1} \operatorname{SUBS} k i l l\right) \wedge\left(s v_{1} \mathrm{AFF} o\right) \rightarrow \exists s v_{2}\left[\left(s v_{2} \operatorname{SUBS} \text { die }\right) \wedge\left(s v_{2} \operatorname{AFF} o\right)\right]
$$

On the other hand, the relationship between causality and temporal order can be expressed by an R-axiom $\left(s_{1}\right.$ CAUS $\left.s_{2}\right) \rightarrow \neg\left(s_{2}\right.$ ANTE $\left.s_{1}\right)$. For an overview of the different types of axioms used in MultiNet see [13].

\subsection{Stratification of the Networks}

The MultiNet formalism was designed such that qualitatively different aspects of meaning will not be pressed into one flat structure. To this end, the nodes and arcs of MultiNet are embedded in a multidimensional space of so-called layer attributes. The layer specifications for arcs are defined by a K-TYPE attribute which assigns one of the knowledge 
Table 2. Relations of preextensional layer. $p(e)^{n}$ denotes extensions of entities with [ETYPE $=n$ ].

\begin{tabular}{lll}
\hline Relation & Signature & Short Characteristics \\
\hline *DIFF & $p(e)^{n} \times p(e)^{n} \rightarrow p(e)^{n}$ & Set difference (of extensions) \\
DPND & $p(e) \times p(e)$ & Dependency (of extensions) \\
ELMT & $p(e)^{n} \times p(e)^{n+1}$ & Membership (of extensions) \\
EXT & $e n t \times p(e)$ & Extension \\
SUBM & $p(e)^{n} \times p(e)^{n}$ & Subsumption (inclusion of extensions) \\
$*$ UNION & $p(e)^{n} \times p(e)^{n} \rightarrow p(e)^{n}$ Set-theoretic union (of extensions) \\
\hline
\end{tabular}

types mentioned in point 5, Section 2.1 to each argument of the edge. For nodes, the layer specification comprises a whole set of so-called layer attributes (see Figure 2). The following layer attributes describe a conceptual entity at the intensional level:

- GENER: The degree of generality indicates whether a conceptual entity is generic (value: $g e$ ) or specific (value: $s p$ ). Example: "A lion $[\mathrm{GENER}=g e]$ is dangerous." vs. "(This lion) $[\mathrm{GENER}=s p]$ is dangerous."

- QUANT: The intensional quantification represents the quantitative aspect of a conceptual entity, i.e. whether it is a singleton (value: one) or a multitude (values: two, several, many, all etc). Within the set of values characterizing multitudes, we discern between fuzzy quantifiers like several, many, most with value [QUANT=fquant] and non-fuzzy quantifiers like $t w o$, three, all with value [QUANT=nfquant]. Example: "(Two dogs) [QUANT=nfquant, $\mathrm{CARD}=2]$ barked."

A bundle of four additional layer features is concerned with the extensional aspect:

- ETYPE: This attribute characterizes the type of extensionality of an entity with values: $n i l$ - no extension (e.g. intension), 0 - individual that is not a set (e.g. Napoleon I), 1 - entity with a set of elements from type [ETYPE=0] as extension (e.g. $\langle$ many emperors $\rangle,\langle$ the dynasty $\rangle$ ), 2 - entity with a set of elements from type $[\mathrm{ETYPE}=1]$ as extension (e.g. $\langle$ many dynasties $\rangle$ ), etc.

- FACT: The facticity attribute, to be discussed in greater detail in Section 5, discerns actually existing objects and situations $[\mathrm{FACT}=$ real $]$ from non-existing objects or situations $[\mathrm{FACT}=$ nonreal $]$ and hypothetically assumed objects or situations which are labeled [FACT=hypo]. Example: "(The girl) [FACT=real] dreamed that (she was crying) $[\mathrm{FACT}=$ hypo]."

- REFER: This attribute specifies the determination of reference, i.e. whether there is a determined object of reference (value: det) or not (value: indet). The REFER attribute is especially important for guiding coreference resolution in a discourse processing or 'assimilation' stage which constructs an integral representation of a whole text from the representations of individual sentences [14].

Example: "(The boy) $[\mathrm{REFER}=$ det $]$ bought ( a toy) $[\mathrm{REFER}=$ indet $]$."

- CARD: We have already met this cardinality attribute in the introduction. As opposed to the intensional quantification expressed by QUANT, the CARD attribute specifies the actual cardinality of the extension of a node.

- VARIA: The variability indicates whether an object is conceptually varying (value: var) - i.e. it is a 'parameterized object' - or if it is a fixed constant (value: con). Example: "(The teacher) [VARIA =con] looked at (every homework) [VARIA=var]." 


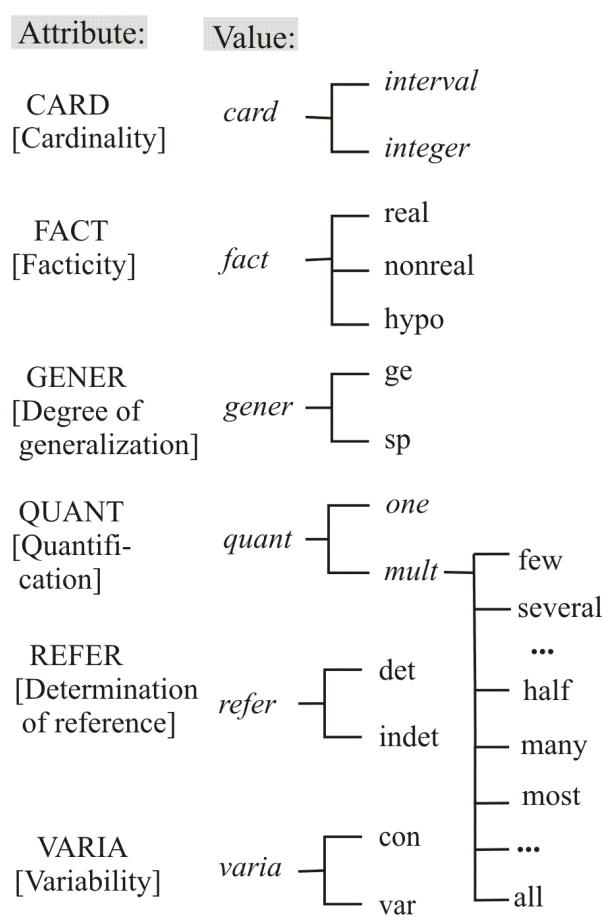

Fig. 2. The multidimensional space of layer attributes

The idea of layers is motivated by an analogy to the mathematics of an $n$-dimensional space. If one fixes a value along one of the axes of an $n$-dimensional coordinate system, one gets a $(n-1)$-dimensional hyperplane. In the same way, if one is fixing one value of a layer attribute (let us assume $[\mathrm{GENER}=g e]$ or $[\mathrm{FACT}=$ hypo]), then one gets a special layer or stratum (in this case the layer of all generic concepts or the layer of all hypothetical entities, respectively). The interplay of these layer attributes becomes visible in the representations of the following two sentences (see Figure 3):
(E1) "All students own bicycles."
(E2) "Every student owns a bicycle."

In Figure 3, the first statement is represented by the POSS arc labeled with (1) and the second by the POSS arc labeled with (2). As shown in the figure, the extension of node $n_{2}$ (representing the concept 〈every student $\rangle$ ) is a varying individual $p_{2}$ within the set $p_{1}$ of all students. The abbreviations $\langle$ all $\rangle$ and $\langle$ every $\rangle$ stand for a whole bundle of layer attributes and their values shown in Table 3. [ETYPE $=+k]$ in this table means that the extension type of a concept characterized with every/all is incremented by $k$ compared to the ETYPE of the basic concept found in the lexicon. The counterpart $p_{3}$ at the preextensional level of node $n_{3}$ at the intensional level (a varying, parameterized, and individual bicycle) is connected by the dependency relation DPND with the extensional representative $p_{2}$ of $n_{2}$. This, together with the layer information of $n_{3}$, gives a special reading to sentence (E2) saying that every single student owns exactly one bicycle de- 


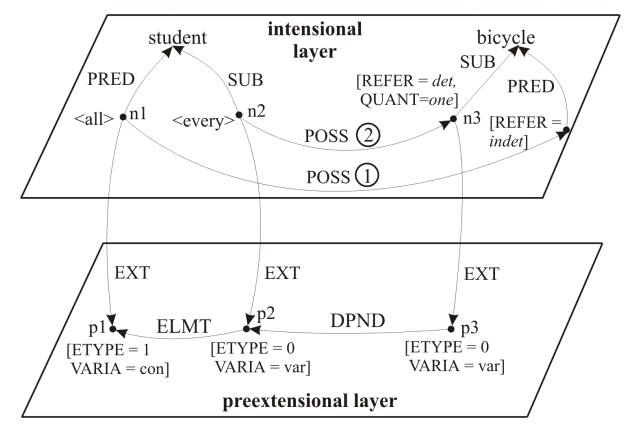

Fig. 3. Sample network with intensional and preextensional layers

Table 3. Detail from the description of quantificators by means of layer attributes

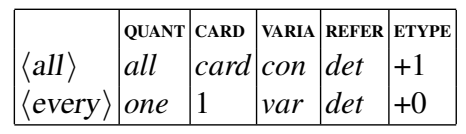

pending on this student. Note that according to Table $3, n_{1}$ has a non-varying collection with determined reference as its extension, while $n_{2}$ and $n_{3}$ have varying extensions with determined and indetermined references, respectively.

\section{Intensional Quantification and Preextensional Cardinality}

The interplay of the intensional and extensional aspects of meaning becomes visible in the linguistic phenomenon of intensional quantification (carried by quantificators ${ }^{2}$ like "several", "most") and their extensional counterparts, the cardinalities of sets.

For the semantic representation of expressions containing these quantificators the combination between the intensional and preextensional layer is needed (see motivating example in introduction). Here we consider a similar case which demonstrates how the example sentences are analyzed on the intensional and preextensional levels:

(S1) "Peter sent his mother many letters." (Node $s v_{1}$ )

(S2) "Fife of them were lost." (Arc $\left.s v_{2}\right)$

The MultiNet represented in Figure 4 reveals that sentence (S1) is indeed represented on the intensional level only. The partitive construction in the second sentence, however, requires a shift to the preextensional level. The representation of (S2) on the right-hand side of the figure asserts that there is a conceptual node on the intensional level which represents a plurality of lost letters. The extension of this node has cardinality 5. Since the partitive "five of them" rather than "all of them" was used, we know that a proper subset relationship holds between the set of lost letters and the letters Peter had sent to his mother. We conclude that the set of sent letters has indeed more than 5 elements, which conforms to the intuitive expectations.

\footnotetext{
${ }^{2}$ To distinguish these constructs from the existential quantifier $\exists$ and the universal quantifier $\forall$ in logics, we deliberately coined the term "quantificator".
} 


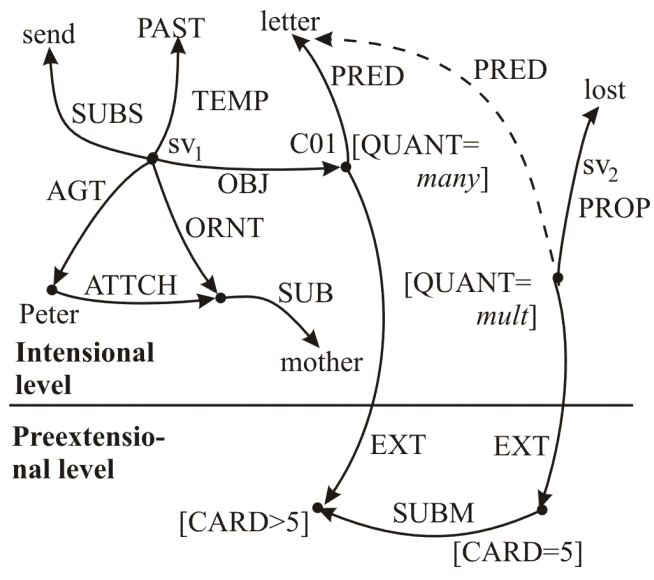

Fig. 4. Intensional quantificators vs. cardinalities on the preextensional level

\section{Different Types of Negation}

The proper modeling of negation in a KRS is a difficult task, since the usual negation of first-order logic does not cover all aspects of linguistic negation. Let us consider the following example:

(N1) "The concert was not unpleasant."

If we assume a constant $c$ for the concrete concert and a unary predicate PLEASANT for the meaning of the adjective "pleasant", the typical way of expressing the meaning of the above proposition in FOL would be: $\neg \neg \operatorname{PLEASANT}(c)$, which is equivalent to $\operatorname{PLEASANT}(c)$. However, PLEASANT $(c)$ is clearly not the meaning of $(\mathrm{N} 1)$, which demonstrates that the law of double negation does not hold for this kind of negated gradable properties. Notice that a degree-based modeling along the lines of [15] might solve this problem on the theoretical side but only at the price of a very complicated representation which is hardly suited for knowledge processing.

MultiNet offers two types of negation to deal with negated sentences. An intensional negation of a state of affairs $s v$ is expressed by a modal restriction ( $s v$ MODL *NON) which corresponds to the negation contained in the sentence itself. But there is also the preextensional negation of $s v$ expressed by the layer attribute $[\operatorname{FACT}(s v)=$ nonreal saying that $s v$ does not hold in reality. To see the rationale for discerning these two kinds of negation, consider the problem of interpreting subordinated sentences introduced by the conjunction "instead of" in the case that the subordinated sentence again involves negation. An example is "Instead of taking nothing from the table, the guest ate everything up" which contains an intensional negation within the subordinated clause by the negator "nothing". This negation expressed by $\left(s v_{2} \mathrm{MODL} * \mathrm{NON}\right)$ in Figure 5 is the basis for answering a question aiming at the expectations of the dialogue partners ("What did you expect?" - "That he took nothing!") However the conjunction "instead of" introduces a second negation as a presupposition, which has to be represented by [FACT=nonreal $]$ with $s v_{2}$, stating that the state of affairs which had been expected does not hold in reality. If we mingled these two types of negation (intensional vs. preexten- 


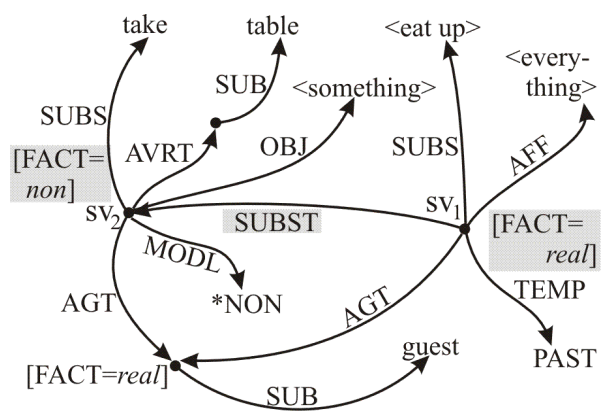

Fig. 5. The two types of negation in subordinated sentences

sional) or even deleted both of them on the basis of a wrongly applied law of double negation we could not answer the question asking for an expectation. However, the two types of negation may obviously cancel out under certain circumstances. This would be the case for questions with a purely factual character ("Did he take something from the table?" - Answer: "Yes!"'). In order to account for these effects, application of the law of double negation (combining factual or preextensional negation with intensional negation) should depend on the type of the question.

\section{Existential Presuppositions and Counterfactuals}

Another aspect of reference that must be captured by a KRS is concerned with the facticity of the entities of interest. In natural language we can discuss the existence or nonexistence of objects of a certain kind - like ghosts, aliens or black holes. It is also possible to talk about situations or events regardless of facticity. Consequently, [6,7] emphasize the importance of modeling non-existing objects for knowledge representation. In predicate logic, however, there is an inherent existence assumption for all individuals (i.e. individual constants) because the model-theoretic semantics demands that all constants denote in the universe. This is why we introduced the attribute FACT to express the facticity of a considered entity. As opposed to certain dialects of modal predicate logic which use an existence predicate to tell existing objects from non-existing ones [5], we discern three possible cases, viz the real, nonreal and hypo as mentioned above. The third value hypo is needed, for instance, to describe states of affairs in conditionals or in opaque contexts. [8] argues that seven ways of existing should be distinguished, which could easily be accomplished by extending the set of facticity values.

Table 4 shows some basic axioms related to facticity, which also express some of the existential presuppositions made while uttering a factual statement. Axiom (a) states that the agent or experiencer of a factual event/state must really exist. For example, Peter can only know something if he is a real person. The same can also be said about LOC and TEMP, i.e. a real situation takes place at a real location and has a temporal extent in the real world. An exception to Axiom (a) is the MCONT role which expresses mental content or modal embedding. Here, somebody's belief in the existence of the Yeti is classified hypo by axiom (b). However, as shown by (c), there are special cases (e.g. knowing something) which license the conclusion that the second argument of MCONT 
Table 4. Selected axioms related to facticity. Axioms marked by $*$ must be considered defaults.
(a) $\left(x \mathrm{R} x^{\prime}\right) \wedge[\operatorname{FACT}(x)=$ real $] \rightarrow\left[\mathrm{FACT}\left(x^{\prime}\right)=\right.$ real $]$ where $R \in\{\mathrm{AFF}, \mathrm{AGT}, \mathrm{EXP}, \mathrm{OBJ}, \ldots\}$ (valid for most argument roles)
$(\mathrm{b})^{*}\left(x \operatorname{MCONT} x^{\prime}\right) \rightarrow\left[\mathrm{FACT}\left(x^{\prime}\right)=\right.$ hypo $]$
(c) $(s \operatorname{SUBS}$ know $) \wedge\left(s\right.$ MCONT $\left.s^{\prime}\right) \wedge[\operatorname{FACT}(s)=$ real $] \rightarrow\left[\operatorname{FACT}\left(s^{\prime}\right)=\right.$ real $]$
(d) $\left(x \mathrm{R} x^{\prime}\right) \wedge[\operatorname{FACT}(x)=$ real $] \rightarrow\left[\operatorname{FACT}\left(x^{\prime}\right)=\right.$ real $]$ where $R \in\{$ PARS, ELMT, HSIT $\}$ (for all meronymy relations)
(e) $\left(s \operatorname{COND} s^{\prime}\right) \rightarrow[\operatorname{FACT}(s)=$ hypo $] \wedge\left[\mathrm{FACT}\left(s^{\prime}\right)=\right.$ hypo

is factual as well. For that reason (b) was marked as default knowledge. Axiom (d) states that a real aggregate can only be composed of real parts. Finally, Axiom (e) expresses the observation that if-then-relationships involve hypothetical situations. Consider the following example: "If John returns late, he will be tired." In this case, there is obviously no commitment as to the facticity of premise and conclusion, which are thus tagged [FACT $=$ hypo $]$.

It should be pointed out that the FACT attribute allows us to model all types of implicative verbs, factive verbs and counterfactive verbs as listed in the classification of [16]. We will pick two examples to demonstrate how to accomplish this.

The verb "forget to" is a so-called two-way implicative whose facticity pattern can be described by the following two axioms:

$$
\begin{aligned}
& (s \mathrm{SUBS}\langle\text { forget to }\rangle) \wedge(s \mathrm{MCONT} m) \wedge[\operatorname{FACT}(s)=\text { real }] \rightarrow[\operatorname{FACT}(m)=\text { nonreal }] \\
& (s \mathrm{SUBS}\langle\text { forget to }\rangle) \wedge(s \mathrm{MCONT} m) \wedge[\operatorname{FACT}(s)=\text { nonreal }] \rightarrow[\operatorname{FACT}(m)=\text { real }] .
\end{aligned}
$$

In other words, if Ed forgets to close the door, then Ed does not close the door; and if Ed does not forget to close the door, then he actually closes the door.

Our second example is "forget that", a factive verb with a positive existential presupposition. Here we only need one additional axiom to describe the facticity pattern:

$$
(s \mathrm{SUBS}\langle\text { forget that }\rangle) \wedge(s \mathrm{OBJ} m) \wedge[\operatorname{FACT}(s)=\text { nonreal }] \rightarrow[\operatorname{FACT}(m)=\text { real }] .
$$

Thus, if Ed does not forget that he closed the door, then Ed closed the door. Due to the use of the OBJ role, the above axiom (a) already captures the opposite case, i.e. if Ed forgets that he closed the door, we may conclude that Ed closed the door.

In practice, the entailments are expressed by axiom schemes for all verbs of the considered type. In this way, all facticity patterns of [16] can be captured.

Counterfactuals are among the most prominent semantic phenomena where the combination of intensional modality and preextensional facticity is needed to represent the full meaning. Figure 6 shows the proposed representation of the sentence: "If the boy had come yesterday, he would have got the toy". Our analysis of counterfactuals introduces two pairs of situations which correspond to the upper and lower half of the depicted MultiNet. There are two hypothetical situations $\tilde{s v}_{1}$ and $\tilde{s v_{2}}$ linked by a conditional, expressing "If the boy came yesterday, he got the toy". Moreover there is a pair of negated factive situations with a causal relationship which roughly express "The boy did not get the toy because he did not come yesterday." These negated factive situations and their causal link are implicit in the counterfactual statement. 


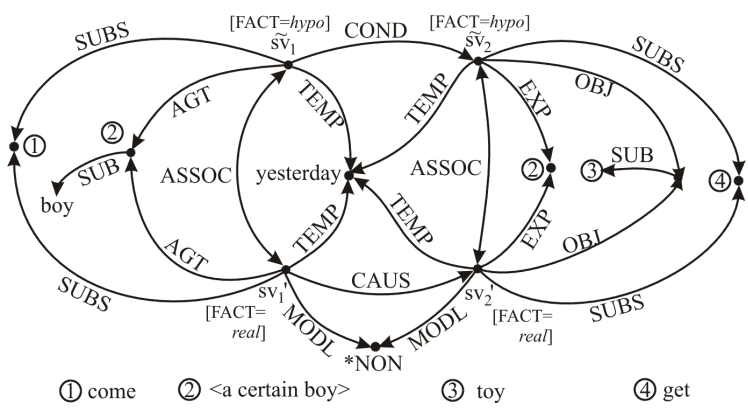

Fig. 6. Representation of a counterfactual

\section{The Role of Extension Types in Semantic Representations}

The extension type of concepts, introduced in Sect. 2.4 and represented by the layer attribute ETYPE, is not only important for defining the signatures of relations and functions at the preextensional level (cf. Table 2). Combined with other expressional means, it also explains the difference between quantified concepts as "all children" with $[\mathrm{ETYPE}=1]$ and "every child" with [ETYPE $=0]$ (cf. Figure 3). While expressions of the first type, when used in a sentence like "All children bought a gift", allow for a cumulative and a distributive reading (and, thus, need background knowledge for a further interpretation), this is not the case for expressions with [ETYPE=0], as in "Every child bought a gift". The latter allows only for a distributive reading.

An effect seldom properly taken into account is the influence of the extension type on the specification of lexical meanings, especially in connection with the selectional restrictions of verbs. Concepts underlying verbs like "die out" or verbal constructs like "be at odds with each other" can only be used either in connection with concepts of [ETYPE $\geq 1]$ or possibly with generic concepts. Examples: "(The Miller clan $)_{[\mathrm{ETYPE}=1]}$ died out." - "(The Blue Whale $)_{[\mathrm{GENER}=g e]}$ has almost died out."

\section{Partitive and Coordinating Quantification}

The last phenomenon to be discussed is the semantic interpretation of partitive and coordinating quantifiers whose semantic representation requires the expressional means of the preextensional layer. Partitive quantifications are typically expressed by prepositions like "besidelexcept", "apart from", "without", and some uses of the preposition "of". However, also coordinations building pluralities like "Some teachers and all students" must refer to operations on sets which belong to the preextensional level. The following example comprises typical effects in one sentence (N2):

"Many students and all teachers of the school except the director cleaned the room." Though such examples are routinely treated in the Theory of Generalized Quantifiers [17], they are not visible in state-of-the art KRS. MultiNet, however, offers a corresponding representation shown in Figure 7: To represent the meaning of sentence (N2) and especially of the phrase "Many students and all teachers of the school except the 


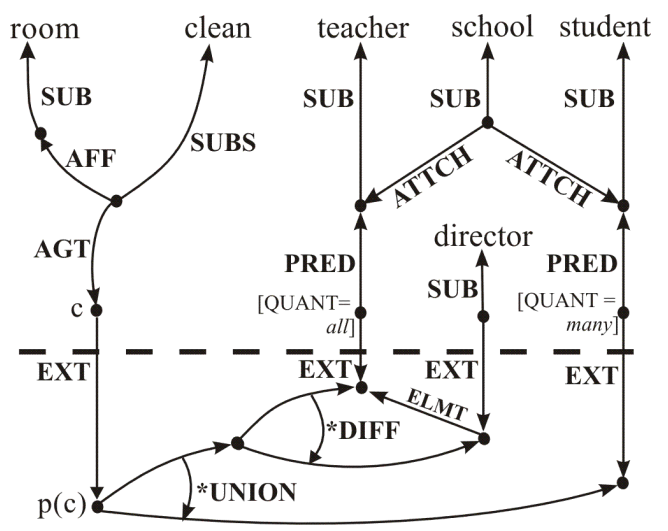

Fig. 7. The construction of pluralities by means of prepositions

director", a concept $c$ at the intensional level and its representative $p(c)$ at the preextensional level have to be created, which are related by $(c \operatorname{EXT} p(c))$. For specifying $p(c)$, at first a set difference is constructed using *DIFF to capture the meaning of "except". After that the union of the result with the set of many students has to be built using the function *UNION to capture the effect of "and" on the coordinating quantification.

\section{Conclusion}

We have argued that a comprehensive meaning representation for natural language expressions must combine the intensional and extensional aspects of semantic description. Since the full extension of a natural language concept is typically out of reach, a preextensional layer was introduced which allows to deal with partially described extensions or 'preextensions'. Using Multilayered Extended Semantic Networks for semantic representation, we discussed several linguistic phenomena whose interpretation arguably involves the extensional aspect. We then explained how to handle theses cases by combining the intensional and preextensional descriptive means of the framework. Obviously there is specialized research into most of these issues (like handling partitive and coordinating quantifications or modeling facticity). However, none of these approaches provides an integrated solution which has made its way into a working KRS. The MultiNet approach, by contrast, has been used as a meaning representation formalism in several projects (e.g. VirWiss [18]). It is also the semantic interlingua for representing user queries in natural language interfaces to internet information providers and to local databases $[19,20]$. Corresponding transformation modules exist which translate MultiNet representations of questions into various target formats like Z39.50 and SQL.

The inference system of MultiNet and its axiom system are used in question answering (QA) systems [21,22], in the natural language interfaces mentioned above, and in the virtual electronic laboratory VILAB [23]. The use of MultiNet in the QA systems InSicht and IRSAW is continually evaluated in the CLEF QA track (see [21,22]) as well as in the answer-validating system MAVE (AVE-track of CLEF [24]). In all CLEF competitions from 2004 to 2006 these systems ranked among the best three for German. 


\section{References}

1. Carnap, R.: Meaning and Necessity. Chicago University Press, Chicago (1947)

2. Janas, J.M., Schwind, C.B.: Extensional Semantic Networks. In Findler, N.V., ed.: Associative Networks. Academic Press (1979) 267-305

3. Brachman, R.J., Schmolze, J.G.: An overview of the KL-ONE knowledge representation system. Cognitive Science 9 (1985) 171-216

4. Baader, F., Calvanese, D., McGuinness, D., Nardi, D., Patel-Schneider, P., eds.: The Description Logics Handbook. Cambridge University Press, Cambridge (2004)

5. Lambert, K., ed.: Philosophical Applications of Free Logic. Oxford University Press (1991)

6. Hobbs, J.: Ontological promiscuity. In: Proc. 23rd Annual Meeting of the ACL. (1985)

7. Shapiro, S.C., Rapaport, W.J.: SNePS considered as a fully intensional propositional semantic network. In Cercone, N., McCalla, G., eds.: The Knowledge Frontier: Essays in the Representation of Knowledge. Springer, New York (1987) 262-315

8. Hirst, G.: Existence assumptions in knowledge representation. Artificial Intelligence 49 (1991) 199-242

9. Bobrow, D., Condoravdi, C., Crouch, R., de Paiva, V., Kaplan, R., Karttunen, L., King, T., Zaenen, A.: A basic logic for textual inference. In: Proceedings of the AAAI Workshop on Inference for Textual Question Answering, Pittsburgh, PA (2005)

10. Helbig, H.: Knowledge Representation and the Semantics of Natural Language. Springer, Berlin (2006)

11. Gnörlich, C.: Technologische Grundlagen der Wissensverwaltung für die automatische Sprachverarbeitung. PhD thesis, FernUniversität Hagen, Hagen, Germany (2002)

12. Hartrumpf, S., Helbig, H., Osswald, R.: The semantically based computer lexicon HaGenLex - Structure and technological environment. Traitement automatique des langues 44 (2003)

13. Helbig, H., Glöckner, I.: Reasoning over semantic networks - a typology of axioms for natural language inference. In: Proc. MRCS-07, Hyderabad, India (2007) 30-34

14. Glöckner, I., Hartrumpf, S., Helbig, H.: Automatic knowledge acquisition by semantic analysis and assimilation of textual information. In: Proc. KONVENS 2006, Konstanz (2006)

15. Pulman, S.G.: Formal and computational semantics: a case study. In: Proc. 7th Int. Workshop on Computational Semantics, Tilburg (2007) 181-196

16. Nairn, R., Condoravdi, C., Karttunen, L.: Computing relative polarity for textual inference. In: Proceedings of ICoS-5 (Inference in Computational Semantics), Buxton, UK (2006)

17. Keenan, E., Moss, L.: Generalized quantifiers and expressive power of natural language. In Benthem, J., Meulen, A., eds.: Generalized Quantifiers in Natural Language. Foris (1984)

18. Glöckner, I., Knoll, A.: Natural-language navigation in multimedia archives: An integrated approach. In: Proc. 7th ACM Multimedia Conference (MM-99), Orlando, FL (1999)

19. Leveling, J., Helbig, H.: Das NLI-Z39.50 - Natürlichsprachliche Informationssuche zur Verbesserung der Literaturrecherche. In: Proc. KONVENS 2006, Konstanz (2006) 97-100

20. Helbig, H., Gnörlich, C., Menke, D.: Realization of a user-friendly access to networked information retrieval systems. Informatik-Bericht 196, FernUniversität Hagen (1996)

21. Hartrumpf, S.: Question answering using sentence parsing and semantic network matching. In: Working Notes for the CLEF 2004 Workshop, Bath, England (2004) 385-392

22. Leveling, J.: A baseline for NLP in domain-specific information retrieval. In: Accessing Multilingual Information Repositories (Proc. CLEF-05). LNCS-4022. Springer (2006)

23. Lütticke, R., Gnörlich, C., Helbig, H.: VILAB - a virtual electronic laboratory for applied computer science. In: Proc. Conf. Networked Learning in a Global Environment. (2002)

24. Glöckner, I.: University of Hagen at QA@ CLEF 2006: Answer validation exercise. In Nardi, A., Peters, C., Vicedo, J.L., eds.: Working Notes for the CLEF 2006 Workshop. (2006) 